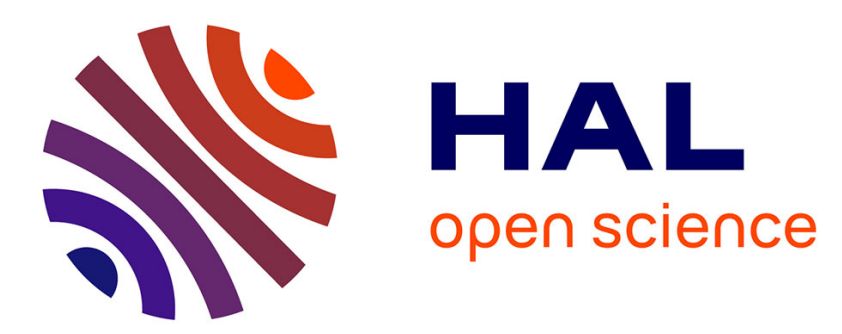

\title{
Boundary Feedback Control of Linear Hyperbolic Systems: Application to the Saint-Venant-Exner Equations
}

Christophe Prieur, Joseph J Winkin

\section{To cite this version:}

Christophe Prieur, Joseph J Winkin. Boundary Feedback Control of Linear Hyperbolic Systems: Application to the Saint-Venant-Exner Equations. Automatica, 2018, 88, pp.44-51. 10.1016/j.automatica.2017.11.028 . hal-01702529

HAL Id: hal-01702529

https://hal.science/hal-01702529

Submitted on 6 Feb 2018

HAL is a multi-disciplinary open access archive for the deposit and dissemination of scientific research documents, whether they are published or not. The documents may come from teaching and research institutions in France or abroad, or from public or private research centers.
L'archive ouverte pluridisciplinaire HAL, est destinée au dépôt et à la diffusion de documents scientifiques de niveau recherche, publiés ou non, émanant des établissements d'enseignement et de recherche français ou étrangers, des laboratoires publics ou privés. 


\title{
Boundary Feedback Control of Linear Hyperbolic Systems: Application to the Saint-Venant-Exner Equations
}

\author{
Christophe Prieur ${ }^{\mathrm{a}} \quad$ Joseph J. Winkin ${ }^{\mathrm{b}}$ \\ ${ }^{\mathrm{a}}$ Univ. Grenoble Alpes, CNRS, Gipsa-lab, F-38000 Grenoble, France \\ ${ }^{\mathrm{b}}$ Namur Center of Complex Systems (naXys) and Department of Mathematics, University of Namur, Belgium
}

\begin{abstract}
Distributed parameter systems modeled by hyperbolic partial differential equations are considered in this paper. The dynamic models include a source term and heterodirectional velocities. A boundary control problem is introduced and it is first shown that it is well-posed (in the sense of Tucsnak and Weiss), under appropriate assumptions coupling the boundary conditions and the source term. Then a sufficient exponential stability condition is derived using operator theory. This condition is written in terms of Linear Matrix Inequalities that are numerically tractable and that allow an optimization program. Connections with another classical stability condition are given. This approach is applied to the Saint-Venant-Exner equation describing the dynamics of the water level, of the water flow and of the sediment inside of a channel. The effect of the friction and of the slope are taken into account in the application model.
\end{abstract}

\section{Introduction}

A class of distributed parameter systems including the shallow water equations as an important application is considered in this paper. There exists a large literature dealing with nonlinear equations, as those developing the analysis of characteristic curves or Lyapunov methods, see [3] for a recent textbook on this subject. In the present paper, the system is described by a linear hyperbolic partial differential equation (PDE) which is assumed to be nonhomogeneous with a control acting on the boundary. This control problem arises in many different contexts such as gas transport [14, traffic control [12] or flow dynamics in open-channels 7 . For these kinds of infinite dimensional systems, many different techniques are available, such as an analysis of the time evolution of the characteristic curves (as done in e.g., [17, 21]), Lyapunov methods (see e.g. [10, 9]) and abstract theory for linear systems (as considered in [19, 5, 25]). In this paper, we will develop some semigroup techniques for a control problem of a system of balance laws with an application to the dynamics of flow and sediments in an open channel, as considered in 8, where an exponentially stabilizing output feedback controller is computed, using a backstepping design. With respect to this recent reference, a fully different method is suggested, since we first solve a general boundary control problem, and then apply its solution to the particular linear hyperbolic system obtained by linearizing the Saint-Venant-Exner equation around a given equilibrium.
First the control problem is shown to be well-posed as a boundary control system in the sense of the book 25]. In particular, it is shown that the dynamics operator is the infinitesimal generator of a strongly continuous $\left(C_{0}\right)$ semigroup of bounded linear operators, which is (exponentially) stable and that the unbounded control operator defining the boundary control action is admissible for this semigroup. Next the stability analysis is performed exploiting some sufficient conditions dealing with the source term of the hyperbolic PDE and with matrix inequalities combining the velocities matrix and the boundary conditions. The advantage of these conditions reside in the fact that they can be rewritten in a numerically tractable form allowing an optimization of the unknown variables. More precisely checking the assumptions of the main result is shown to be equivalent to solving Linear Matrix Inequalities (LMI's) which are numerically tractable conditions (see Remark 1 below just before the first main result, namely Theorem 1). Moreover the obtained sufficient condition is linked with classical Lyapunov function based sufficient conditions for stability analysis and boundary stabilization (as done in particular in [7]).

The obtained sufficient exponential stability condition is used to design output feedback boundary controllers for hyperbolic systems of balance laws. Other techniques are available for the design of boundary controllers, as the backstepping techniques (see [26] and references therein). The design method is shown to be numeri- 
cally tractable and it is applied on the linearization of the Saint-Venant-Exner equation at an equilibrium. Using numerical values that are already considered in the literature 16, 20, 11 in a physical sense, we show how to solve our numerically tractable conditions and how to compute a stabilizing output feedback boundary controller. A numerical simulation is also performed to illustrate the obtained exponential stability by means of time-evolution of the three components of the state.

The paper is organized as follows. In Section 2 the model is introduced and some preliminary computations are performed. Section 3 contains the main results on the well-posedness of the control problem and on a sufficient condition for the stability of the open-loop system. Section 4 contains an application in hydraulics. The results are illustrated and some simulations are performed. Section 5 collects concluding remarks and points out some possible research lines.

\section{Description and analysis of the model}

Let us consider a distributed parameter system that is described by the following linear partial differential equation (PDE) model:

$$
\xi_{t}+\Lambda \xi_{x}=M \xi, \quad x \in(0,1), t \geq 0,
$$

where $\Lambda$ is a constant diagonal matrix in $\mathbb{R}^{n \times n}$ of the following form $\Lambda=\left[\begin{array}{cc}\Lambda_{+} & 0 \\ 0 & -\Lambda_{-}\end{array}\right]$, where $\Lambda_{+}=\operatorname{diag}\left(\lambda_{i}\right)_{i=1}^{m} \in \mathbb{R}^{m \times m}$ and $\Lambda_{-}=$ $\operatorname{diag}\left(-\lambda_{i}\right)_{i=m+1}^{n} \in \mathbb{R}^{(n-m) \times(n-m)}$ are positive definite, i.e. $\lambda_{i}>0$ for $i=1, \cdots, m$ and $\lambda_{i}<0$ for $i=m+1, \cdots, n$, where $M$ is a constant matrix in $\mathbb{R}^{n \times n}$ and

$\xi=\xi(x, t)=\left[\begin{array}{l}\xi_{+}(x, t) \\ \xi_{-}(x, t)\end{array}\right]$ where $\xi_{+} \in \mathbb{R}^{m}$ and $\xi_{-} \in \mathbb{R}^{n-m}$

stands for the state. This model is complemented by the boundary condition:

$$
\left[\begin{array}{c}
\xi_{+}(0, t) \\
\xi_{-}(1, t)
\end{array}\right]=K\left[\begin{array}{l}
\xi_{+}(1, t) \\
\xi_{-}(0, t)
\end{array}\right]+N u(t)
$$

where $K$ is a constant matrix in $\mathbb{R}^{n \times n}$ of the following form $K=\left[\begin{array}{ll}0 & K_{0} \\ K_{1} & 0\end{array}\right]$, where $K_{0} \in \mathbb{R}^{m \times(n-m)}$ and $K_{1} \in$ $\mathbb{R}^{(n-m) \times m}$ and $N$ is a full rank matrix in $\mathbb{R}^{n \times p}$ of the following form $N=\left[N_{0}^{\top} N_{1}^{\top}\right]^{\top}$, where $p \leq n, N_{0} \in$ $\mathbb{R}^{m \times p}, N_{1} \in \mathbb{R}^{(n-m) \times p}$ and $u(t) \in \mathbb{R}^{p}$ is the control at time $t \geq 0$, to be designed.
With a view to studying the well-posedness of system (1)-(3), let us introduce the following operator:

$$
L \xi=-\Lambda \xi^{\prime}+M \xi,
$$

where' stands for the space derivative. Consider also the complex Hilbert spaces $Z=H^{1}\left((0,1) ; \mathbb{C}^{n}\right)$ and $X=$ $L^{2}\left((0,1) ; \mathbb{C}^{n}\right)$. Recall that $Z \subset X$ with continuous embedding. Observe that $L$ is a bounded linear operator from $Z$ to $X$, i.e. $L \in \mathcal{L}(Z, X)$. In addition, consider the time-invariant operator $G \in \mathcal{L}\left(Z, \mathbb{C}^{n}\right)$ given by, for all $\xi \in Z$,

$$
G \xi=\left[\begin{array}{l}
\xi_{+}(0) \\
\xi_{-}(1)
\end{array}\right]-K\left[\begin{array}{l}
\xi_{+}(1) \\
\xi_{-}(0)
\end{array}\right]
$$

and the Hilbert subspace $X_{1}$ of $Z$ which is defined by

$$
X_{1}:=\operatorname{ker} G=\left\{\xi \in Z:\left[\begin{array}{l}
\xi_{+}(0) \\
\xi_{-}(1)
\end{array}\right]=K\left[\begin{array}{l}
\xi_{+}(1) \\
\xi_{-}(0)
\end{array}\right]\right\} .
$$

Finally, in the analysis below, we will also need the restriction of the operator $L$ to $X_{1}$, denoted by $A$ :

$$
A:=L_{\mid X_{1}} .
$$

Observe that $A \in \mathcal{L}\left(X_{1}, X\right)$.

Preliminary remark: The stationary solution of the PDE (1) with initial condition $\xi(0)$, i.e. the solution of the ordinary differential equation

$$
-\Lambda \frac{d \xi}{d x}+M \xi=0, x \in[0,1]
$$

is given by $\xi(x)=\exp \left(\Lambda^{-1} M x\right) \xi(0)$, for all $x$ in $[0,1]$. With this computation in mind, we may rewrite the boundary condition (3). To be more specific, given $c=$ $\left[\begin{array}{l}c_{+} \\ c_{-}\end{array}\right]$in $\mathbb{C}^{n}$, the boundary condition

$$
\left[\begin{array}{l}
\xi_{+}(0) \\
\xi_{-}(1)
\end{array}\right]-K\left[\begin{array}{l}
\xi_{+}(1) \\
\xi_{-}(0)
\end{array}\right]=c
$$

is satisfied if and only if

$$
\begin{aligned}
& {\left[\begin{array}{l}
\xi_{+}(0) \\
\left(\exp \left(\Lambda^{-1} M\right) \xi(0)\right)_{-}
\end{array}\right]} \\
& \quad-\left[\begin{array}{ll}
0 & K_{0} \\
K_{1} & 0
\end{array}\right]\left[\begin{array}{l}
\left(\exp \left(\Lambda^{-1} M\right) \xi(0)\right)_{+} \\
\xi_{-}(0)
\end{array}\right]=\left[\begin{array}{l}
c_{+} \\
c_{-}
\end{array}\right],
\end{aligned}
$$

or equivalently

$$
\left[\begin{array}{lc}
I_{m} & -K_{0} \\
{\left[-K_{1}\right.} & \left.I_{n-m}\right] \\
\cdot \exp \left(\Lambda^{-1} M\right)
\end{array}\right] \cdot \xi(0)=c .
$$


In order that the initial state $\xi(0)$ be uniquely determined by the latter equation, i.e. in order to convert the two-point boundary value problem (5)-(6) into a Cauchy problem, the following auxiliary result is useful, resulting from a direct application of Schur complement:

Lemma 1 The matrix

$$
\left[\begin{array}{lc}
I_{m} & -K_{0} \\
{\left[-K_{1}\right.} & \left.I_{n-m}\right] \cdot \exp \left(\Lambda^{-1} M\right)
\end{array}\right] \quad \text { is invertible }
$$

if and only if so is the matrix

$$
\left[\begin{array}{ll}
-K_{1} & I_{n-m}
\end{array}\right] \cdot \exp \left(\Lambda^{-1} M\right) \cdot\left[\begin{array}{c}
K_{0} \\
I_{m}
\end{array}\right]
$$

\section{Well-posedness and stabilization}

\subsection{Well-posedness}

As an intermediary step towards the well-posedness property, let us consider the following auxiliary boundary condition:

$$
\left[\begin{array}{l}
\xi_{+}(0, t) \\
\xi_{-}(1, t)
\end{array}\right]=K\left[\begin{array}{l}
\xi_{+}(1, t) \\
\xi_{-}(0, t)
\end{array}\right]+\tilde{N} \tilde{u}(t)
$$

where the matrix $\tilde{N}:=[N V] \in \mathbb{R}^{n \times n}$ and the submatrix $V \in \mathbb{R}^{n \times(n-m)}$ is chosen such that $\tilde{N}$ is nonsingular and where $\tilde{u}(t) \in \mathbb{R}^{n}$ is an auxiliary input. The wellposedness of the control problem is solved in the next proposition.

Proposition 1 If the matrix given by (8) is invertible, then the system described by the PDE (1) with the boundary condition (9) is a boundary control system, in the sense of [25, Chap. 10], which is described by the pair of operators $(L, \tilde{G})$, where $\tilde{G}:=\tilde{N}^{-1} G$, or equivalently by the abstract differential equation $\xi_{t}=L \xi$ with $\tilde{G} \xi=\tilde{u}$.

Proof. First observe that without loss of generality $\tilde{N}$ is the identity matrix, whence $\tilde{G}=G$. Now recall that $L \in \mathcal{L}(Z, X)$ and observe that $G \in \mathcal{L}\left(Z, \mathbb{C}^{n}\right)$. Moreover these operators satisfy the properties characterizing a boundary control system (see [25, Definition 10.1.1] where the constant $\beta$ is set to zero). Indeed:

a) By Lemma 1, thanks to invertibility of the matrix in (8), for every $c$ in $\mathbb{C}^{n}$ the equation $G \xi=c$ admits a (unique) solution $\xi \in Z$. It follows that $G$ is onto.

b) It follows also from condition (7) that the operator $L$ restricted to $X_{1}=\operatorname{ker} G$ is onto, since for every $f \in X$, the equation $L \xi=f, \quad \xi \in X_{1}$ is equivalent to an ordinary differential equation having a solution $\xi$ which is uniquely determined by $\xi(0)$ and $f$.

c) Moreover ker $L \cap \operatorname{ker} G=\{0\}$ (this follows from the arguments used in the previous item with $f=0$ ).

d) Finally, $X_{1}=\operatorname{ker} G$ is dense in $X$, since it contains a dense subspace of $X=L^{2}\left((0,1) ; \mathbb{C}^{n}\right)$, namely $H_{0}^{1}\left((0,1) ; \mathbb{C}^{n}\right)$.

To get that the PDE (1) with the boundary condition (3) is well-posed, recalling the definition (4) of $A$, it remains to check that the control operator defined in the boundary condition (3) defines an operator $B$ which is an admissible control operator (following the terminology of [25, Definition 10.1.1]). To do that, let us first compute $B^{\star}$. For all $z$ in $Z$ and for all $\Psi$ in $\mathcal{D}\left(B^{\star}\right)$, it holds $\langle L z, \Psi\rangle-\left\langle z, A^{\star} \Psi\right\rangle=\left\langle G z, N B^{\star} \Psi\right\rangle$. Thus

$$
\begin{gathered}
\left\langle z(0)-K z(1), N B^{\star} \Psi\right\rangle \\
=\left\langle-\Lambda z^{\prime}+M z, \Psi\right\rangle-\left\langle z, \Lambda \Psi^{\prime}+\overline{M^{\top}} \Psi\right\rangle \\
=-\left\langle\Lambda z^{\prime}, \Psi\right\rangle-\left\langle z, \Lambda \Psi^{\prime}\right\rangle+\langle M z, \Psi\rangle-\left\langle z, \overline{M^{\top}} \Psi\right\rangle \\
=-\langle\Lambda z(1), \Psi(1)\rangle+\langle\Lambda z(0), \Psi(0)\rangle \\
=\langle K z(1), K \Lambda \Psi(0)\rangle+\langle z(0), \Lambda \Psi(0)\rangle
\end{gathered}
$$

by using an integration by parts, and by using that $\Psi$ in $\mathcal{D}\left(A^{\star}\right)$ (and thus $\left.\Lambda \Psi(1)=\overline{K^{\top}} \Lambda \Psi(0)\right)$.

Therefore $\langle z(0)-K z(0), \Lambda \Psi(0)\rangle=\left\langle z(0)-K z(0), N B^{\star} \Psi\right\rangle$ and thus $B^{\star} \Psi=\overline{N^{\top}} \Lambda \Psi(0)$.

The control operator $B: U \rightarrow X_{-1}$ is defined by, for all $\xi$ in $U, B \xi=\delta_{0} \Lambda N \xi$ where $\delta_{0}$ is the Dirac function at $x=0$. $B$ is an admissible control operator following [25. Definition 4.2.1], since with $M=0$, this control operator is admissible (see e.g. [25, Example 4.2.7] for the scalar case), and a bounded perturbation $M \neq 0$ does not render $B$ not admissible.

Therefore the PDE (1) with the boundary condition (3) is well-posed and with [25, Prop. 10.1.8], we get

Proposition 2 If the matrix given by (8) is invertible then, for every $T>0, \xi(0)$ in $Z$ and $u \in \mathcal{H}^{1}((0, T) ; U)$ which satisfies the compatibility condition $G \xi(0)=$ $N u(0)$, the PDE (1) with the boundary condition (3) has a unique solution $\xi$ and $\xi \in C([0, T] ; Z) \cap C^{1}([0, T] ; X)$.

\subsection{Stability analysis}

In the sequel, it will be assumed that the following two conditions hold and it will be shown that they ensure the stability of the boundary control system introduced in Proposition 1 above. Each of these conditions is twofold, 
involving a "primal" condition on (the parameters defining) the operator $A$ and a "dual" condition on the adjoint operator $A^{*}$. It turns out that the two parts of each of these conditions are actually equivalent: see Remark 1c below.

Assumption 1 There exists a diagonal positive definite matrix $P$ in $\mathbb{R}^{n \times n}$ such that

$$
P M+M^{*} P \leq 0,
$$

where $M^{*}:=\overline{M^{\top}}$ denotes the Hermitian conjugate of the matrix $M$, and equivalently there exists a diagonal positive definite matrix $Q$ in $\mathbb{R}^{n \times n}$ such that

$$
Q M^{*}+M Q \leq 0
$$

Assumption 2 The matrices $\Lambda$ and $K$ are such that

$$
\left\|(|\Lambda| P)^{1 / 2} K(|\Lambda| P)^{-1 / 2}\right\|<1
$$

and

$$
\left\|\left(Q|\Lambda|^{-1}\right)^{-1 / 2} K\left(Q|\Lambda|^{-1}\right)^{1 / 2}\left[\begin{array}{ll}
0 & I_{m} \\
I_{n-m} & 0
\end{array}\right]\right\|<1,
$$

where $P$ is a diagonal positive definite matrix which satisfies inequality (10), $Q$ is a diagonal positive definite matrix which satisfies inequality (11), $\|$.$\| denotes the ma-$ trix norm induced by the usual euclidean vector norm, $|\mathcal{M}|$ denotes the absolute value of the matrix $\mathcal{M}$, i.e. its entries are the absolute values of those of $\mathcal{M}$ and, in particular, $|\Lambda|=\left[\begin{array}{cc}\Lambda_{+} & 0 \\ 0 & \Lambda_{-}\end{array}\right]$.

Remark 1 a) Conditions (12) and (13) are trivially satisfied whenever the boundary feedback gain matrix $K$ is the null matrix. However this choice is clearly not always feasible in applications: this is the case notably for the application that is studied in Section 4 .

b) In Assumption 1, the existence of a diagonal positive definite matrix $P$ satisfying inequality (10) is guaranteed whenever the matrix $M$ is Lyapunov stable with entries having the same sign. This is the case for the application that is studied in Section 4. In addition, the matrix $Q$ satisfying inequality (11) can be selected to be $Q=P^{-1}$. However (many) other choices are possible. See also Section 4.

c) Conditions (10) and (12) are equivalent to the following one:

$$
\rho(K):=\inf _{D \in \mathcal{D}}\left\|(|\Lambda| D)^{1 / 2} K(|\Lambda| D)^{-1 / 2}\right\|<1,
$$

where $\mathcal{D}$ is the set of all diagonal positive definite matrices $D$ such that $D M+M^{*} D \leq 0$. This condition is exactly the same as the one that is used in [7, Theorem 2, p.111] and that is obtained by means of a Lyapunov function. Similarly, conditions (11) and (13) are equivalent to

$$
\begin{gathered}
\tilde{\rho}(K):= \\
\inf _{\tilde{D} \in \tilde{\mathcal{D}}}\left\|\left(\tilde{D}|\Lambda|^{-1}\right)^{-1 / 2} K\left(\tilde{D}|\Lambda|^{-1}\right)^{1 / 2}\left[\begin{array}{ll}
0 & I_{m} \\
I_{n-m} & 0
\end{array}\right]\right\| \\
<1,
\end{gathered}
$$

where $\tilde{\mathcal{D}}$ is the set of all diagonal positive definite matrices $\tilde{D}$ such that $\tilde{D} M^{*}+M \tilde{D} \leq 0$.

Conditions (14) and (15) can be seen as sub-optimization problems, that can be solved numerically for $D$ and $\tilde{D}$, with a given fixed gain $K$, by using algorithmic methods for the resolution of LMIs (as introduced e.g. in 4, 23). As shown in the proof of the next theorem, these conditions can be stated under the form of LMI's. More precisely, condition (12) is equivalent to

$$
K|\Lambda|^{-1} W K^{*}<|\Lambda|^{-1} W,
$$

where $W=P^{-1}$, and condition (13) is equivalent to

$$
K Q|\Lambda|^{-1} K^{*}<Q|\Lambda|^{-1} \text {. }
$$

From the previous discussion, one may conclude that the existence of a diagonal positive definite matrix $P$ such that conditions (10) and (16) hold is equivalent to the existence of a diagonal positive definite matrix $Q$ such that conditions (11) and (17) hold. In other words, Assumptions 1 and 2 are equivalent to any of the following assumptions.

Assumption 3 There exists a diagonal positive definite matrix $P$ such that (10) and (16) hold.

Assumption 4 There exists a diagonal positive definite matrix $Q$ such that (11) and (17) hold.

Note that computing diagonal positive definite matrices $P$ and $Q$, such that Assumptions 3 or 4 hold, are two convex conditions in the unknown variables $P$ and $Q$ which can be solved e.g. on Matlab using YALMIP [18]. This convex problem in solved when designing a stabilizing feedback in Section 4.3 below.

Theorem 1 If the matrix in (8) is invertible and under Assumption 3 (or equivalently under Assumption 4), the operator $A$ generates an exponentially stable $C_{0}$ semigroup $T(t)$ of bounded linear operators on $X$, and there exists $\nu>0$ such that, for all $t \geq 0,\|T(t)\| \leq$ $\exp (-\nu t)$. 
Before proving Theorem 1, let us connect this result with 22 as done in the following remark.

Remark 2 Combining Assumption 1 and condition (14) yields a set of assumptions that is implied by 22 , Assumption 3.8, Page 664] when restricting to spaceinvariant operators. Thus Theorem 1 can be seen as a generalization of [22, Theorem 3.9, Page 665]. By item c) of Remark 1, Assumptions 1 and 2 are equivalent to the conditions of [3, Proposition 5.2]. Therefore we recover the stability conditions of $A$ using a completely different approach (namely a dissipativity approach versus a Lyapunov function approach in 3]), which generalizes the conditions of [22, Section 3].

Proof. The proof of Theorem 1 is split into 4 steps.

1) Closedness of the operator $A$.

First observe that, by the proof of Proposition 1, the linear operator $A$, whose domain is $X_{1}=\operatorname{ker} G$, is densely defined on $X$, onto and invertible with a bounded linear inverse $A^{-1}$, which is given for every $f \in X$ by

$$
\begin{aligned}
A^{-1} f= & \exp \left(\Lambda^{-1} M x\right)\left[\begin{array}{ll}
I_{m} & -K_{0} \\
{\left[-K_{1}\right.} & \left.I_{n-m}\right] \cdot \exp \left(\Lambda^{-1} M\right)
\end{array}\right]^{-1} \\
& {\left[\begin{array}{l}
0 \\
I(1)_{-}-K_{1} I(1)_{+}
\end{array}\right]-I(x) }
\end{aligned}
$$

where

$$
I(x)=\int_{0}^{x} \exp \left(\Lambda^{-1} M(x-z)\right) \Lambda^{-1} f(z) d z
$$

i.e. 0 is in the resolvent set $\rho(A)$ of $A$. It follows by 25 , Remark 2.2.4, p. 24], or more specifically by [5. Theorem A.3.46, p. 596], that the operator $A$ is closed.

\section{2) Adjoint $A^{\star}$ of the operator $A$}

The adjoint $A^{\star}$ is given for all $\psi$ in its domain

$$
\mathcal{D}\left(A^{\star}\right)=\left\{\psi \in Z: K^{*}|\Lambda|\left[\begin{array}{l}
\psi_{+}(0) \\
\psi_{-}(1)
\end{array}\right]=|\Lambda|\left[\begin{array}{l}
\psi_{+}(1) \\
\psi_{-}(0)
\end{array}\right]\right\},
$$

by

$$
A^{\star} \psi=\Lambda \psi^{\prime}+M^{*} \psi
$$

Indeed, it suffices to observe that the usual pairing identity $\left\langle A^{\star} \psi, z\right\rangle=\langle\psi, A z\rangle$ holds for all $z \in \mathcal{D}(A)$ and for all $\psi \in \mathcal{D}\left(A^{\star}\right)$. To check this, let us consider the operator
$A^{\star}$ given by (21) on its domain (20) and observe that, for all $z \in \mathcal{D}(A)$ and for all $\psi \in \mathcal{D}\left(A^{\star}\right)$,

$$
\begin{aligned}
\left\langle A^{\star} \psi, z\right\rangle= & \int_{0}^{1} \psi^{\prime \star} \Lambda^{\star} z d x+\int_{0}^{1}\left(M^{\star} \psi\right)^{\star} z d x \\
= & \int_{0}^{1} \psi_{+}^{\prime \star} \Lambda_{+} z_{+} d x-\int_{0}^{1} \psi_{-}^{\prime \star} \Lambda_{-} z_{-} d x \\
& +\int_{0}^{1} \psi^{\star} M z d x \\
= & -\int_{0}^{1} \psi_{+}^{\star} \Lambda_{+} z_{+}^{\prime} d x+\left.\psi_{+}^{\star} \Lambda_{+} z_{+}\right|_{0} ^{1} \\
& +\int_{0}^{1} \psi_{-}^{\star} \Lambda_{-} z_{-}^{\prime} d x-\left.\psi_{-}^{\star} \Lambda_{-} z_{-}\right|_{0} ^{1} \\
& +\int_{0}^{1} \psi^{\star} M z d x
\end{aligned}
$$

where an integration by part has been performed in the first two integrals. Now, using the definitions of $\mathcal{D}\left(A^{\star}\right)$ and $K$, we get, for all $\psi \in \mathcal{D}\left(A^{\star}\right)$,

$$
\left[\begin{array}{ll}
0 & K_{1}^{\star} \\
K_{0}^{\star} & 0
\end{array}\right]\left[\begin{array}{l}
\Lambda_{+} \psi_{+}(0) \\
\Lambda_{-} \psi_{-}(1)
\end{array}\right]=\left[\begin{array}{l}
\Lambda_{+} \psi_{+}(1) \\
\Lambda_{-} \psi_{-}(0)
\end{array}\right]
$$

which is equivalent to

$$
\left[\begin{array}{c}
K_{1}^{\star} \Lambda_{-} \psi_{-}(1) \\
K_{0}^{\star} \Lambda_{+} \psi_{+}(0)
\end{array}\right]=\left[\begin{array}{c}
\Lambda_{+} \psi_{+}(1) \\
\Lambda_{-} \psi_{-}(0)
\end{array}\right]
$$

Combining the boundary conditions (23) with (22) and the definitions of $A$ and its domain $\mathcal{D}(A)$ yields the following identity, for all $z \in \mathcal{D}(A)$ and for all $\psi \in \mathcal{D}\left(A^{\star}\right)$ :

$$
\begin{aligned}
\left\langle A^{\star} \psi, z\right\rangle= & \langle\psi, A z\rangle+\psi_{+}^{\star}(1) \Lambda_{+} z_{+}(1) \\
& -\psi_{+}^{\star}(0) \Lambda_{+} K_{0} z_{-}(0)-\psi_{+}^{\star}(1) \Lambda_{+} z_{+}(1) \\
& +\psi_{+}^{\star}(0) \Lambda_{+} K_{0} z_{-}(0) \\
= & \langle\psi, A z\rangle .
\end{aligned}
$$

Thus the adjoint of $A$ is given by (20) and (21).

In addition, let us now check that it follows from Assumptions 1 and 2, i.e. from the inequalities (10), (11), (12) and (13), that

(i) there exists $\nu_{1}>0$, such that, for all $z$ in $X_{1}$, $\Re\langle A z, P z\rangle \leq-\nu_{1}|z|_{X}^{2}$, hence the operator $A$ is dissipative, and

(ii) the operator $A^{\star}$ is also dissipative, such that there exists $\nu_{2}>0$ satisfying, for all $\psi$ in $\mathcal{D}\left(A^{\star}\right), \Re\left\langle A^{\star} \psi, Q \psi\right\rangle \leq$ $-\nu_{2}|\psi|_{X}^{2}$. 


\section{3) Dissipativity of the operator $A$}

In order to check the fact (i), note that, for all $z$ in $X_{1}$,

$$
\begin{aligned}
\Re\langle A z, P z\rangle & =\Re \int_{0}^{1}-\left(\Lambda z^{\prime}\right)^{\star} P z d x+\Re \int_{0}^{1}(M z)^{\star} P z d x \\
& \leq \Re \int_{0}^{1}-z^{\star \star} \Lambda P z d x,
\end{aligned}
$$

where (11) from Assumption 1 has been used. Observe that, thanks to the fact that the matrices $\Lambda$ and $P$ are diagonal, $\Lambda P=P \Lambda$ and $\frac{d}{d x}\left(z^{\star} \Lambda P z\right)=2 z^{\prime \star} \Lambda P z$. It follows that

$$
\begin{aligned}
\Re\langle A z, P z\rangle \leq & -\left.\frac{1}{2} z^{\star} \Lambda P z\right|_{0} ^{1} \\
\leq & -\frac{1}{2}\left(z_{+}^{\star}(1) \Lambda_{+} P_{+} z_{+}(1)-z_{-}^{*}(1) \Lambda_{-} P_{-} z_{-}(1)\right. \\
& \left.-z_{+}^{*}(0) \Lambda_{+} P_{+} z_{+}(0)+z_{-}^{*}(0) \Lambda_{-} P_{-} z_{-}(0)\right) .
\end{aligned}
$$

By using the definition of $\mathcal{D}(A)$, we obtain that, for all $z$ in $\mathcal{D}(A), z_{+}(0)=K_{0} z_{-}(0)$ and $z_{-}(1)=K_{1} z_{+}(1)$. By plugging these boundary conditions in the second and third terms of the right-hand side of the inequality above, we get

$$
\begin{gathered}
\Re\langle A z, P z\rangle \\
\leq-\frac{1}{2}\left(z_{+}^{\star}(1) \Lambda_{+} P_{+} z_{+}(1)-z_{+}^{\star}(1) K_{1}^{*} \Lambda_{-} P_{-} K_{1} z_{+}(1)\right. \\
\left.-z_{-}(0)^{*} K_{0}^{*} \Lambda_{+} P_{+} K_{0} z_{-}(0)+z_{-}^{*}(0) \Lambda_{-} P_{-} z_{-}(0)\right)
\end{gathered}
$$

and thus $\Re\langle A z, P z\rangle \leq \frac{1}{2} v^{\star} E v$ with $v^{\top}=\left[z_{+}(1), z_{-}(0)\right]$ and the matrix

$$
E=\left[\begin{array}{ll}
-\Lambda_{+} P_{+}+K_{1}^{\star} \Lambda_{-} P_{-} K_{1} & 0 \\
0 & K_{0}^{\star} \Lambda_{+} P_{+} K_{0}-\Lambda_{-} P_{-}
\end{array}\right] .
$$

Now note that (12) in Assumption 2 is equivalent to the LMI

$$
K|\Lambda|^{-1} P^{-1} K^{\star}<|\Lambda|^{-1} P^{-1}
$$

and using the definition of $K$ and the coordinate decomposition (2), it is also equivalent to

$$
\left\{\begin{array}{c}
K_{1}^{*} \Lambda_{-} P_{-} K_{1}<\Lambda_{+} P_{+} \\
K_{0}^{*} \Lambda_{+} P_{+} K_{0}<\Lambda_{-} P_{-}
\end{array}\right.
$$

Therefore, under (12) in Assumption 2, reinterpreted as (24), there exists a positive value $\nu_{1}$ such that, for all $z$ in $\mathcal{D}(A), \Re\langle A z, P z\rangle \leq-\nu_{1}\left(\left|z_{+}(1)\right|^{2}+\left|z_{-}(0)\right|^{2}\right)$ and, by the boundedness of the trace operator from $Z$ to $\mathbb{R}$ with norm equal to 1 , it follows that, for all $z$ in $\mathcal{D}(A)$, $\Re\langle A z, P z\rangle \leq-\nu_{1}|z|_{X}^{2}$. Moreover, since $P$ is symmetric positive definite, there exists $\tilde{\nu}_{1}>0$ such that, for all $z$ in $\mathcal{D}(A), \Re\langle A z, z\rangle \leq \tilde{\nu}_{1} \Re\langle A z, P z\rangle$. Thus, with a suitable $\hat{\nu}_{1}>0$, for all $z$ in $\mathcal{D}(A)$,

$$
\Re\langle A z, z\rangle \leq-\hat{\nu}_{1}|z|_{X}^{2} .
$$

In other words, $A$ is a dissipative operator.

4) Dissipativity of the operator $A^{\star}$

To check the fact (ii), let us compute, for all $\psi$ in $\mathcal{D}\left(A^{\star}\right)$,

$$
\begin{aligned}
\Re\left\langle A^{\star} \psi, Q \psi\right\rangle & =\Re \int_{0}^{1}\left(\Lambda \psi^{\prime}\right)^{\star} Q \psi d x+\Re \int_{0}^{1}\left(M^{\star} \psi\right)^{\star} Q \psi \\
& \leq \Re \int_{0}^{1} \psi^{\prime \star} \Lambda Q \psi d x,
\end{aligned}
$$

where (10) from Assumption 1 has been used. Note that, thanks to the commutativity property $\Lambda Q=Q \Lambda$, there holds $\frac{d}{d x}\left(\psi^{\star} \Lambda Q \psi\right)=2 \psi^{\prime \star} \Lambda Q \psi$ and thus

$$
\begin{gathered}
\Re\left\langle A^{\star} \psi, Q \psi\right\rangle \leq\left.\frac{1}{2} \psi^{\star} \Lambda Q \psi\right|_{0} ^{1} \\
\leq \frac{1}{2}\left(\psi_{+}^{\star}(1) \Lambda_{+} Q_{+} \psi_{+}(1)-\psi_{-}^{\star}(1) \Lambda_{-} Q_{-} \psi_{-}(1)\right. \\
\left.-\psi_{+}^{\star}(0) \Lambda_{+} Q_{+} \psi_{+}(0)+\psi_{-}^{\star}(0) \Lambda_{-} Q_{-} \psi_{-}(0)\right) \\
\leq \frac{1}{2}\left(\psi_{-}^{\star}(1) \Lambda_{-} K_{1} Q_{+} \Lambda_{+}^{-1} K_{1}^{\star} \Lambda_{-} \psi_{-}(1)\right. \\
-\psi_{-}^{\star}(1) \Lambda_{-} Q_{-} \psi_{-}(1)-\psi_{+}^{\star}(0) \Lambda_{+} Q_{+} \psi_{+}(0) \\
\left.+\psi_{+}^{\star}(0) \Lambda_{+} K_{0} Q_{-} \Lambda_{-}^{-1} K_{0}^{\star} \Lambda_{+} \psi_{+}(0)\right)
\end{gathered}
$$

where (23) has been used in the first and the last terms. Therefore

$$
\Re\left\langle A^{\star} \psi, Q \psi\right\rangle \leq \frac{1}{2} w^{\star} F w
$$

with $w^{\top}=\left[\psi_{+}(0), \psi_{-}(1)\right]$ and the matrix

$$
F=\left[\begin{array}{ll}
\Lambda_{+} K_{0} Q_{-} \Lambda_{-}^{-1} K_{0}^{\star} \Lambda_{+}-\Lambda_{+} Q_{+} & 0 \\
0 & \Lambda_{-} K_{1} Q_{+} \Lambda_{+}^{-1} K_{1}^{\star} \Lambda_{-}-\Lambda_{-} Q_{-}
\end{array}\right] .
$$

Now note that (13) in Assumption 2 is equivalent to

$$
\begin{aligned}
& \left(Q|\Lambda|^{-1}\right)^{-1 / 2} K\left(Q|\Lambda|^{-1}\right)^{1 / 2}\left[\begin{array}{ll}
0 & I_{m} \\
I_{n-m} & 0
\end{array}\right] \\
& {\left[\begin{array}{ll}
0 & I_{m} \\
I_{n-m} & 0
\end{array}\right]\left(Q|\Lambda|^{-1}\right)^{1 / 2} K^{\star}\left(Q|\Lambda|^{-1}\right)^{-1 / 2}<I_{n}}
\end{aligned}
$$

and, by using the commutativity of $\Lambda$ and $Q$, it is also equivalent to

$$
\left(Q|\Lambda|^{-1}\right)^{-1 / 2} K Q|\Lambda|^{-1} K^{\star}\left(Q|\Lambda|^{-1}\right)^{-1 / 2}<I_{n} .
$$

Finally by pre- and post-multiplying the last inequality by $(Q|\Lambda|)^{1 / 2}$, one gets the LMI: $|\Lambda| K Q|\Lambda|^{-1} K^{\star}|\Lambda|<$ 
$Q|\Lambda|$, i.e., by using the definition of $K$ and the coordinate decomposition (2),

$$
\left\{\begin{array}{c}
\Lambda_{+} K_{0} Q_{-} \Lambda_{-}^{-1} K_{0}^{\star} \Lambda_{+}<\Lambda_{+} Q_{+} \\
\Lambda_{-} K_{1} Q_{+} \Lambda_{+}^{-1} K_{1}^{\star} \Lambda_{-}<\Lambda_{-} Q_{-} .
\end{array}\right.
$$

Therefore, under (13) in Assumption 2, reinterpreted as (26), the following inequality holds, for all $\psi$ in $\mathcal{D}\left(A^{\star}\right)$ : $\Re\left\langle A^{\star} \psi, Q \psi\right\rangle \leq-\nu_{2}\left(\left|\psi_{+}(0)\right|^{2}+\left|\psi_{-}(1)\right|^{2}\right)$, for some positive constant $\nu_{2}$, and, by the continuity of the trace operator from $Z$ to $\mathbb{R}$, it follows that, for all $\psi$ in $\mathcal{D}\left(A^{\star}\right)$, $\Re\left\langle A^{\star} \psi, Q \psi\right\rangle \leq-\nu_{2}|\psi|_{X}^{2}$. Moreover, since $Q$ is symmetric positive definite, there exists $\tilde{\nu}_{2}>0$ such that, for all $\psi$ in $\mathcal{D}\left(A^{\star}\right), \Re\left\langle A^{\star} \psi, \psi\right\rangle \leq \tilde{\nu}_{2} \Re\left\langle A^{\star} \psi, Q \psi\right\rangle$. Thus, with a suitable $\hat{\nu}_{2}>0$, for all $\psi$ in $\mathcal{D}\left(A^{\star}\right)$,

$$
\Re\left\langle A^{\star} \psi, \psi\right\rangle \leq-\hat{\nu}_{2}|\psi|_{X}^{2} .
$$

Then one can conclude that $A^{\star}$ is a dissipative operator.

In view of the facts (i) and (ii), applying Lumer-Phillips Theorem, the operator $A$ is the infinitesimal generator of an $C_{0}$-semigroup $T(t)$ on $X$. Moreover, by (25) and (27), applying [5, Corollary 2.2.3, p. 33], there exists $\nu>0$ satisfying, for all $t \geq 0,\|T(t)\| \leq \exp (-\nu t)$.

Corollary 1 Under the conditions of Theorem 1, the operator $A$ has a pure point spectrum $\sigma(A)=\sigma_{p}(A)$ that is included in the closed left half-plane $\mathbb{C}_{\omega_{0}-}:=\{s \in \mathbb{C}$ : $\left.\Re s \leq \omega_{0}\right\}$ contained in the open left half-plane, where the spectral bound $\omega_{0}:=\sup \Re \sigma(A)<0$ is the growth constant of the $C_{0}$-semigroup generated by $A$. Moreover, any complex number $\lambda$ is an eigenvalue of $A$ iff it is a solution of the characteristic equation:

$\chi(\lambda):=\operatorname{det}\left[\begin{array}{lc}I_{m} & -K_{0} \\ {\left[-K_{1}\right.} & \left.I_{n-m}\right] \\ {\left[\exp \left(\Lambda^{-1}(M-\lambda I)\right)\right.}\end{array}\right]=0$.

Proof. It suffices to observe that any complex number $\lambda$ is in the resolvent set $\rho(A)$ of the operator $A$ iff the matrix

$$
\left[\begin{array}{lc}
I_{m} & -K_{0} \\
{\left[-K_{1}\right.} & \left.I_{n-m}\right] \cdot \exp \left(\Lambda^{-1}(M-\lambda I)\right)
\end{array}\right]
$$

is invertible. In addition, in this case, the resolvent operator is given by

$$
\begin{aligned}
& (A-\lambda I)^{-1} f=\exp \left(\Lambda^{-1}(M-\lambda I) x\right) \\
& {\left[\begin{array}{lc}
I_{m} & -K_{0} \\
{\left[-K_{1}\right.} & \left.I_{n-m}\right] \cdot \exp \left(\Lambda^{-1}(M-\lambda I)\right)
\end{array}\right]^{-1}} \\
& \\
& {\left[\begin{array}{l}
0 \\
I(1, \lambda)_{-}-K_{1} I(1, \lambda)_{+}
\end{array}\right]-I(x, \lambda)}
\end{aligned}
$$

where

$$
I(x, \lambda)=\int_{0}^{x} \exp \left(\Lambda^{-1}(M-\lambda I)(x-z)\right) \Lambda^{-1} f(z) d z,
$$

and is therefore compact. The pure point spectrum property follows by [5. Lemma A.4.19, p. 616].

Moreover, observe that, for every $\lambda=s+\omega$, where $\omega>$ $\omega_{0},(A-\lambda I)^{-1}$ is in the Hardy space $\mathcal{H}^{\infty}(\mathcal{L}(X))$. Recall also that $A$ is the generator of an exponentially stable $C_{0}$-semigroup, hence, by [5. Theorem 5.1.6, p. 223], $A$ satisfies the spectrum determined growth assumption with $\omega_{0}<0$.

\subsection{Design of a stabilizing controller}

Theorem 1 is instrumental to design stabilizing output feedback laws. To be more precise define the output observations as the following part of the state, for all $t \geq 0$,

$$
y(t)=\left[\xi_{+}(1, t), \xi_{-}(0, t)\right]^{\top} .
$$

Then the next corollary follows readily from Proposition 2 and Theorem 1:

Corollary 2 For any matrix $O=\left[O_{0}, O_{1}\right]$ in $\mathbb{C}^{p \times n}$ such that Im $O_{0} \subset \operatorname{Ker} N_{0}$ and Im $O_{1} \subset K e r N_{1}$, define the following output feedback controller by, for all $t \geq 0$,

$$
u(t)=O y(t) .
$$

If the matrix in (8) is invertible, and under Assumptions 1 and 2, where the matrix $K$ has been replaced by $K+N O$ in (8), (12) and (13), there exist a positive value $\nu$ such that it holds, for all $t \geq 0$,

$$
\|\xi(t)\|_{X} \leq \exp (-\nu t)\|\xi(0)\|_{X}
$$

along the solutions to (1) with the boundary condition (3).

\section{Application in hydraulics}

\subsection{Nonlinear and linearized dynamics}

In this section, we are using a system of balance laws by taking into account the potential distributed loss/increase of energy, of momentum... Several examples may come in mind such as the Euler equations. Here we consider the shallow water equations describing the dynamics of the water level, of the water flow and of the sediment inside of a channel. The effect of the friction and of the slope modifies the dynamics and makes a system of balance laws (instead of a system of conservation laws without friction and slope). In this paper we focus on space-invariant equilibrium of Saint-Venant-Exner 
model. For further results on non-constant stationary state of the Saint-Venant-Exner model, see [15.

More specifically, following [13, 16] (see also [7, 20, 8]), a linearization around a space invariant equilibrium could be performed to obtain the following model in Riemann coordinates

$$
\xi_{t}+\Lambda \xi_{x}=M \xi
$$

where $\xi=\left(\xi_{1}, \xi_{2}, \xi_{3}\right)^{\top}, \Lambda(x)=\operatorname{diag}\left(\lambda_{1}, \lambda_{2}, \lambda_{3}\right)$, and, for all $x \in[0,1], t \geq 0$,

$$
M(x)=\left(\begin{array}{ccc}
\alpha_{1} & \alpha_{2} & \alpha_{3} \\
\alpha_{1} & \alpha_{2} & \alpha_{3} \\
\alpha_{1} & \alpha_{2} & \alpha_{3}
\end{array}\right),
$$

with $\alpha_{k}=\left(3 \mathcal{V}^{\star}-2 \lambda_{k}\right) \theta_{k}$ for suitable $\theta_{k} \in \mathbb{R}, k=1,2,3$. It is proved in [16] that, for all $k=1,2,3, \alpha_{k}<0$ (and thus the matrix in (31) is stable, and a possible diagonal definite positive matrix $P$ such that $M^{\top} P+P M<0$ is $\left.P=\operatorname{diag}\left(-\alpha_{1},-\alpha_{2},-\alpha_{3}\right)\right)$. Therefore Assumption 1 holds.

Moreover in the same references, assuming positive flow, it is noted the three eigenvalues of $\Lambda$ satisfy

$$
0<\lambda_{1}<\lambda_{2}, \lambda_{3}<0
$$

Our approach could be adapted to the case of a negative flow.

\subsection{Boundary conditions}

The boundary conditions of (30) are defined by hydraulic control devices such as pumps and valves. Here it is assumed that the water levels are measured at both ends of the open channel, and that the control action can be directly prescribed by the control devices. More precisely, in the Riemann coordinates, we consider the following set of boundary conditions, given three tuning parameters $k_{13}, k_{31}, k_{32}$ to be defined (they are tuning control variables)

$$
\left(\begin{array}{l}
\xi_{1}(0, t) \\
\xi_{2}(0, t) \\
\xi_{3}(1, t)
\end{array}\right)=K\left(\begin{array}{l}
\xi_{1}(1, t) \\
\xi_{2}(1, t) \\
\xi_{3}(0, t)
\end{array}\right)
$$

where

$$
K=\left(\begin{array}{ccc}
0 & 0 & k_{13} \\
0 & 0 & \eta\left(k_{13}\right) \\
k_{31} & k_{32} & 0
\end{array}\right),
$$

and $\eta$ is a nonlinear function, depending on the equilibrium under consideration, see 20 for an expression. See the same reference to check that these controllers depend only on the outputs that are observed.

\subsection{Design of a stabilizing feedback}

Following the notations of Section 2 , we let $m=2, n=3$, $K_{0}=\left(k_{13} \eta\left(k_{13}\right)\right)^{\top}$ and $K_{1}=\left(k_{31} k_{32}\right)$. In the boundary conditions, we do not consider any control actions (thus the control operator is admissible), but we now aim at computing the tuning gains $k_{13}, k_{31}$ and $k_{32}$ so that Corollary 2 applies, yielding a boundary controller such that the closed-loop system is exponentially convergent.

To do that, let us consider the following numerical values $\lambda_{1}=7.72 \times 10^{-4}, \lambda_{2}=13$ and $\lambda_{3}=-10$ with the equilibrium considered in [16, 20, 11.

We may check that by letting $k_{13}=0.05, k_{31}=0$ and $k_{32}=-0.8, P=\operatorname{diag}\left(-\alpha_{1},-\alpha_{2},-\alpha_{3}\right)$, and $Q=$ $\operatorname{diag}\left(\alpha_{2} \alpha_{3}, \alpha_{1} \alpha_{3}, \alpha_{1} \alpha_{2}\right)$ it holds

$$
\operatorname{det}\left[\left(-K_{1} 1\right) \exp \left(\Lambda^{-1} M\right)\left(K_{0} 1\right)\right] \neq 0,
$$

and Equations (12) and (13) hold where $|\Lambda|=$ $\operatorname{diag}\left(\lambda_{1}, \lambda_{2},\left|\lambda_{3}\right|\right)$. Moreover it holds $P M+\overline{M^{\top}} P \leq 0$ and $M Q+Q \overline{M^{\top}} \leq 0$. Thus, the matrix given by (8) is invertible and Assumptions 1 and 2 hold. Therefore Corollary 2 applies with $O=0$.

Other choices are possible for $P$ and $Q$, as already discussed in Remark 1. As an example, we may let $Q=$ $P^{-1}$, and check that $P M+\overline{M^{\top}} P \leq 0$ as soon as $M Q+$ $Q \overline{M^{\top}} \leq 0$.

Let us illustrate the asymptotic stability by means of numerical simulations ${ }^{1}$ The time evolutions of the first, second and third components of the solution to (30) and (33) starting from the initial conditions $\xi(x, t=0)=$ $(\cos (4 \pi x)-1, \cos (2 \pi x)-1,-\cos (4 \pi x)+1)$ for all $x$ in $(0,1)$ are shown in Figures 1-3 where it is checked that the solution converges to the origin. The boundary conditions (33) with the tuned gains $k_{13}, k_{31}$ and $k_{32}$ are given in Figure 4.

Note that, due to the very small value of $\left|\lambda_{1}\right|$ with respect to the absolute values of the other velocities $\left|\lambda_{2}\right|$ and $\left|\lambda_{3}\right|$, it is checked that the first component converges to the origin more slowly than the other components. Compare Figure 1 with Figures 2 and 3 where a different scale for the time $t$ is used. This is consistent with the theory of singularly perturbed hyperbolic systems as studied in 24 .

\section{Conclusion}

Hyperbolic systems described by balance laws have been considered in this paper. A boundary control problem

\footnotetext{
1 The simulation codes can be downloaded from http://www.gipsa-lab.fr/ christophe.prieur/Codes/ automatica17.zip
} 


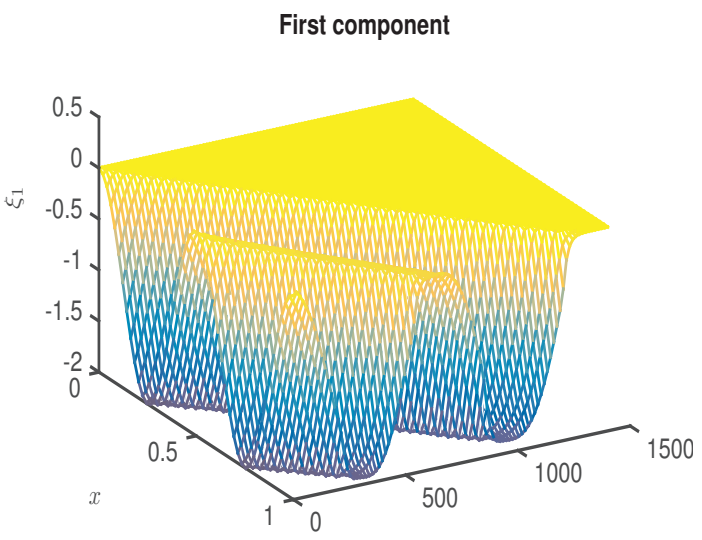

Figure 1. Time evolution of the first component of the $\xi$-solution.

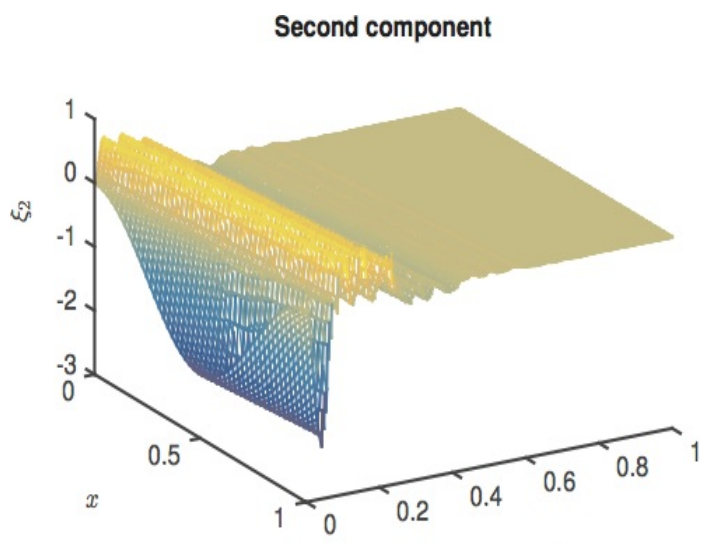

Figure 2. Time evolution of the second component of the $\xi$-solution.

Third component

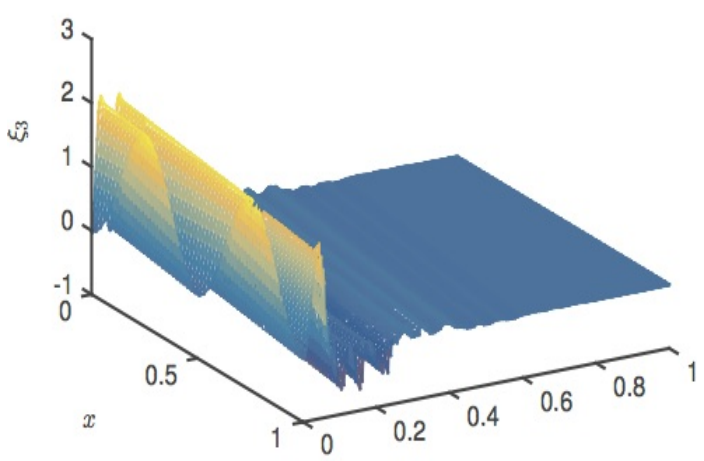

Figure 3. Time evolution of the third component of the $\xi$-solution.

has been studied using semigroup theory. The main results suggest a new sufficient condition for the wellposedness of the boundary control problem and for the stability of the closed-loop system. The sufficient conditions are written in terms of matrix inequalities that are related to by-now classical Lyapunov theory for hy-

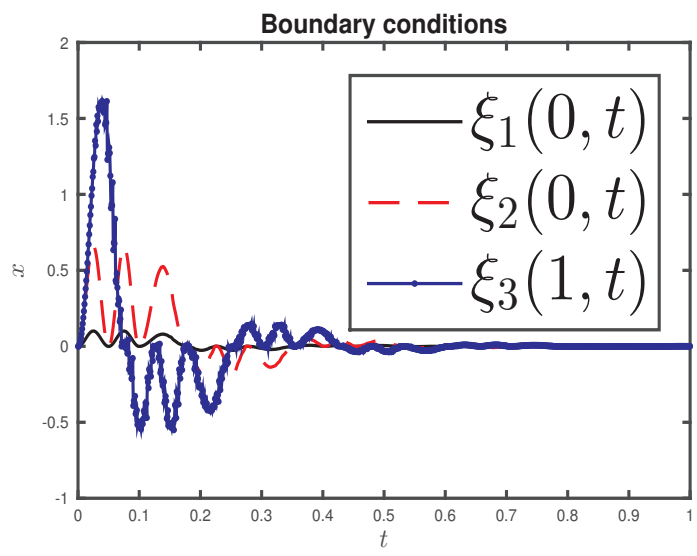

Figure 4. Time evolution of the boundary conditions (33) when applying the tuned gains $k_{13}, k_{31}$ and $k_{32}$.

perbolic systems as developed in e.g., 10, 9. As an illustration of the main results, the Saint-Venant-Exner equation describing the dynamics of the flow and of the sediments inside of an open-channel were considered. Some simulations have been performed to approximate the time evolution of the solutions.

This study leaves many open questions open. In particular, the linear-quadratic (LQ) optimal control problem is a natural extension of this work, using the framework of [27]. It could be fruitful to adapt the techniques of 25 to derive the Riccati equation and to design an LQ optimal control. An analogous (but different) control problem has been already solved in 1 and 2 , where a linearquadratic optimal control is computed for a class of firstorder hyperbolic nonlinear partial differential equations. See also 6] where approximate boundary observation is used to compute an LQ optimal controller. Finally it could be interesting to study the effect of the disturbances in the Saint-Venant-Exner equation on the closed-loop system performance.

\section{Acknowledgement}

This paper presents research results of the Belgian network DYSCO (Dynamical Systems, Control and Optimization), funded by the Interuniversity Attraction Poles Programme, initiated by the Belgian state, Science Policy Office (BELSPO). The scientific responsibility rests with its authors.

This work was supported by the PHC Tournesol FR 2012, project 27310WK, and by HYCON2 Network of Excellence "Highly-Complex and Networked Control Systems", grant agreement 257462.

\section{References}

[1] I. Aksikas, A. Fuxman, J.F. Forbes, and J.J. Winkin. LQ control design of a class of hyperbolic 
PDE systems: Application to fixed-bed reactor. $A u$ tomatica, 45(6):1542-1548, 2009.

[2] I. Aksikas, J.J. Winkin, and D. Dochain. Optimal lq-feedback control for a class of first-order hyperbolic distributed parameter systems. ESAIM: Control, Optimisation and Calculus of Variations, 14(04):897-908, 2008.

[3] G. Bastin and J.-M. Coron. Stability and Boundary Stabilization of 1-D Hyperbolic Systems, volume 88 of Progress in Nonlinear Differential Equations and Their Applications. Springer, 2016.

[4] S. P. Boyd, L. El Ghaoui, E. Feron, and V. Balakrishnan. Linear matrix inequalities in system and control theory, volume 15. SIAM, 1994.

[5] R. Curtain and H. Zwart. An introduction to infinite-dimensional linear systems theory. Springer-Verlag, 1995.

[6] J. R. Dehaye and J. Winkin. LQ-optimal boundary control of infinite-dimensional systems with Yosidatype approximate boundary observation. Automatica, 67:94-106, 2016.

[7] A. Diagne, G. Bastin, and J.-M. Coron. Lyapunov exponential stability of 1-D linear hyperbolic systems of balance laws. Automatica, 48(1):109-114, 2012.

[8] A. Diagne, M. Diagne, S. Tang, and M. Krstic. Backstepping stabilization of the linearized SaintVenant-Exner model. Automatica, 76:345-354, 2017.

[9] M. Dick, M. Gugat, and G. Leugering. Classical solutions and feedback stabilization for the gas flow in a sequence of pipes. Networks and Heterogeneous Media, 5(4):691-709, 2010.

[10] V. Dos Santos, G. Bastin, J.-M. Coron, and B. d'Andréa Novel. Boundary control with integral action for hyperbolic systems of conservation laws: stability and experiments. Automatica, 44(5):1310 1318, 2008.

[11] V. Dos Santos and C. Prieur. Boundary control of open channels with numerical and experimental validations. IEEE Transactions on Control Systems Technology, 16(6):1252-1264, 2008.

[12] M. Garavello and B. Piccoli. Traffic flow on networks. American institute of mathematical sciences Springfield, 2006.

[13] W.H. Graf. Hydraulics of sediment transport. Water Resources Publications, Highlands Ranch, Colorado, USA, 1984.

[14] M. Gugat, M. Dick, and G. Leugering. Gas flow in fan-shaped networks: classical solutions and feedback stabilization. SIAM Journal on Control and Optimization, 49(5):2101-2117, 2011.

[15] M. Gugat and G. Leugering. Global boundary controllability of the saint-venant system for sloped canals with friction. Annales de l'Institut Henri Poincare (C) Non Linear Analysis, 26(1):257-270, 2009.

[16] J. Hudson and P.K. Sweby. Formulations for numerically approximating hyperbolic systems governing sediment transport. Journal of Scientific Computing, 19:225-252, 2003.

[17] T.-T. Li. Global classical solutions for quasilinear hyperbolic systems, volume 32 of $R A M$ : Research in Applied Mathematics. Masson, Paris, 1994.

[18] J. Löfberg. Yalmip : A toolbox for modeling and optimization in MATLAB. In Proc. of the CACSD Conference, Taipei, Taiwan, 2004.

[19] R. S. Phillips. Dissipative operators and hyperbolic systems of partial differential equations. Transactions of the American Mathematical Society, 90(2):193-254, 1959.

[20] C. Prieur and F. Mazenc. ISS-Lyapunov functions for time-varying hyperbolic systems of balance laws. Mathematics of Control, Signals, and Systems, 24(1):111-134, 2012.

[21] C. Prieur, J. Winkin, and G. Bastin. Robust boundary control of systems of conservation laws. Mathematics of Control, Signals, and Systems, 20(2):173197, 2008.

[22] D. L. Russell. Controllability and stabilizability theory for linear partial differential equations: recent progress and open questions. Siam Review, 20(4):639-739, 1978.

[23] C. Scherer and S. Weiland. Linear matrix inequalities in control. Lecture Notes. Dutch Institute for Systems and Control, Delft, The Netherlands, 2000.

[24] Y. Tang, C. Prieur, and A. Girard. Tikhonov theorem for linear hyperbolic systems. Automatica, $57: 1-10,2015$.

[25] M. Tucsnak and G. Weiss. Observation and Control for Operator Semigroups. Birkhäuser, Basel, 2009.

[26] R. Vazquez and M. Krstic. Boundary control of coupled reaction-advection-diffusion systems with spatially-varying coefficients. IEEE Transactions on Automatic Control, to appear, 2017.

[27] M. Weiss and G. Weiss. Optimal control of stable weakly regular linear systems. Mathematics of Control, Signals, and Systems, 10:287-330, 1997. 